\title{
EVALUACIÓN DE LA ESCALA BORG DE ESFUERZO PERCIBIDO APLICADA A LA REHABILITACIÓN CARDIACA
}

\author{
Natalie Burkhalter*
}

BURKHALTER, N. Evaluación de la escala Borg de esfuerzo percibido aplicada a la rehabilitación cardiaca. Rev.latino-am.enfermagem, Ribeirão Preto, v.4, n.3, p.6573, dezembro 1996.

La escala Borg de esfuerzo percibido mide la gama entera del esfuerzo que el individuo percibe al hacer ejercicio. Esta escala da criterios para hacerle ajustes a la intensidad de ejercicio, o sea, a la carga de trabajo, y así pronosticar y dictaminar las diferentes intensidades del ejercicio en los deportes y en la rehabilitación médica (BORG, 1982). También se puede usar tanto en al atletismo, en la astronáutica, la industria y ambientes militares, como en las situaciones cotidianas. El concepto del esfuerzo percibido es una valoración subjetiva que indica la opinión del sujeto respecto a la intensidad del trabajo realizado (MORGAN, 1973). El sujeto que hace el ejercicio debe asignar un número del 1 al 20, para representar la sensación subjetiva de la cantidad de trabajo desempeñado. La escala es una herramienta valiosa dentro del ámbito del desempeño humano, en que a menudo la consideración importante no es tanto "lo que haga el individuo" "sino" "lo que cree que hace" (MORGAN, 1973).

TÉRMINOS CLAVES: Escala Borg de esfuerzo percibido, rehabilitación cardiaca, rehabilitación por medio del ejercicio

La escala Borg de esfuerzo percibido mide la gama entera del esfuerzo que el individuo percibe al hacer ejercicio. Esta escala da criterios para hacerle ajustes a la intensidad de ejercicio, o sea. A la carga de trabajo, y así pronosticar y

${ }^{*} R N, M S N, C S, C C R N$ - Texas A \& M Internacional University - Escuela de Enfermería Laredo, Texas, U.S.A. 
dictaminar las diferentes intensidades del ejercicio en los deportes y en la rehabilitación médica (BORG, 1982). También se puede usar tanto en el atletismo, en la astronáutica, la industria y ambientes militares, como en las situaciones cotidianas. El concepto del esfuerzo percibido es una valoración subjetiva que indica la opinión del sujeto respecto a la intensidad del trabajo realizado (MORGAN, 1973). El sujeto que hace el ejercicio debe designar un número, del 1 al 20, para representar la sensación subjetiva de la cantidad de trabajo desempeñado. La escala es una herramienta valiosa dentro del ámbito del desempeño humano, en que a menudo la consideración importante no es tanto "lo que haga el individuo"sino"lo que cree que hace"(MORGAN, 1973).

\section{ANTECEDENTES}

La importancia de la escala Borg de esfuerzo percibido en el ejercicio estriba en su vínculo estrecho con los factores que indican la fatiga relativa. La escala (ver el Cuadro 1) tiene unos 30 años de aplicarse en los laboratorios de evaluación de ejercicio, pero en los últimos diez años se ha popularizado más en el ambiente clínico (POLLOCK \& FILMORE, 1991). Se puede usar la escala de Borg para cualquier persona que haga ejercicio, pero no se debe ni usar ni interpretar en el vacío (POLLOCK \& FILMORE, 1991). Dice Borg: "No es una escala perfecta y se debe utilizar acompañado del sentido común y de otros datos pertinentes de tipo clínico, psicológico y fisiológico" (POLLOCK \& FILMORE, 1991).

La escala Borg de esfuerzo percibido se diseñó para ser usada con todo individuo, sin distingo de antecedentes culturales. Más bien, la diversidad cultural ha enriquecido la aplicación de la escala al ser traducida a varios idiomas, incrementando así la población objeto del estudio. Entre los idiomas a los cuales se ha traducido, se encuentran el español, el francés, el alemán, el japonés y el ruso (1982). El primer departamento de Medicina Interna en Yokojama, Kanagawa, Japón utilizó la escala para investigar si en un grupo de mujeres el entrenamiento de los músculos de inspiración alteraba su esfuerzo respiratorio durante el ejercicio (SUZUKI et al., 1993).

WILSON et al. (1993) aplicaron la escala en Londres para estudiar en pacientes normales a nivel del mar, el efecto de la altitud de residencia sobre su percepción de falta de aliento. Originalmente, BORG (1982) afirmó que la escala se podría aplicar por igual a la "mayoría" de las personas, sin distingo de sexo, edad, circunstancias o procedencia nacional. Sin embargo, MORGAN (1973) señala las excepciones en el caso de los ancianos o de los individuos neurasténicos, ansiosos, 
deprimidos o que tengan dificultad para elaborar a nivel mental su percepción de la intensidad del esfuerzo. Las investigaciones han demostrado que hay problemas en ciertos grupos, como por ejemplo en los niños pequeños (MAHON \& MARSH, 1992).

La escala Borg de esfuerzo percibido fue diseñada y altamente recomendada para definir cuándo terminar un ejercicio (GORDON \& GIBBONS, 1990). LA AMERICAN HEART ASSOCIATION (1992) le da amplio uso a la escala con el fin de medir la intensidad del trabajo durante la rehabilitación cardiaca en cada una de sus fases. En la primera fase de esta rehabilitación los ejercicios se ejecutan dentro del ambiente hospitalario. Pero en la Segunda fase se dan en un plantel de consulta externa, dentro de un programa organizado o estructurado. La tercera fase se realiza en el hogar, como por ejemplo, en un programa de caminata, o en un programa de vigilancia propia (GORDON \& GIBBONS, 1990). Así esta herramienta ha demostrado su adaptabilidad para ser usada tanto en el hospital como en hogar y en la consulta externa.

\section{CONFIABILIDAD Y VALIDEZ}

Se han diseñado varios estudios con el fin de comprobar la validez y la confiabilidad de la escala Borg de esfuerzo percibido. Según SKINNER et al. (1973), la consistencia interna se determinó al usar un coeficiente alfa entre el ritmo cardíaco y la percepción subjetiva del esfuerzo, lo cual presentaba una gama desde 0,77 hasta $0,90(\mathrm{~N}=16)$. Según GORDON \& GIBBONS (1990), se observaron correlaciones importantes entre el esfuerzo percibido y la percepción somática: siendo la correlación promedio de 0,75 y de 0,83 , una vez logrado un ritmo estable $(\mathrm{N}=88)$. POLLOCK \& FILMORE (1991), correlacionaron varios factores fisiológicos como el ritmo cardíaco, los niveles de lactato y los niveles de oxígeno al expirar, lo cual les dio una correlación múltiple de 0,85. Los coeficientes de confiabilidad para las áreas entre las pistas fueron computados utilizando la fórmula de correlación producto-momento de Pearson. Cuando BLOEM et al. (1991) usaron la escala Borg, ejecutaron una valoración de confiabilidad usando el "test-retest" $(r=0,77)$, o sea, por prueba y repetición de prueba.

Según SKINNER et al. (1973), se definió la validez utilizando una técnica de doble vía para el análisis de la varianza, y las correlaciones producto-momento de Pearson entre los datos de la media para cada prueba. MORGAN (1973) definió la validez de contenido, la validez del "construct" (andamiaje de conceptos y procedimientos prácticos), y la validez con referencia a criterios, utilizando los siguientes instrumentos: 
Eysenck Personality Inventory (Inventario de Personalidad de Eysenck)

Spielberger's State-Trait Inventory (Inventario de Estado y Cualidad de Spielberger)

Somatic Perception Questionnaire (Cuestionario de Percepción Somática)

Lubin's Depression Adjective Checklist (Registro de Adjetivos de Depresión, de Lubin)

Dichos instrumentos contenían el análisis de factores (el "construct"), las preguntas abiertas (el contenido) y la predicción del nivel de funcionamiento (el criterio).

\section{APLICACIÓN CLÍNICA}

La escala Borg de esfuerzo percibido se ha usado principalmente para evaluar la "tensión" subjetiva experimentada durante el ejercicio dinámico (DUNBAR et al., 1992). En la actualidad, algunos centros la utilizan para ayudar a regular la intensidad del entrenamiento por medio de ejercicio. La escala también se puede relacionar a nivel lineal con el ritmo cardiaco durante la ergometría de bicicleta, la ergometría de brazo, el caminar y el correr (DUNBAR et al., 1992). Su utilidad la ha demostrado principalmente cuando al sujeto se le ha presentado cierta intensidad de ejercicio y se le ha pedido tasar su esfuerzo.

La rehabilitación cardiaca es una de las formas más singulares de ejercicio que aplica la escala Borg de esfuerzo percibido. Este tipo de rehabilitación le ofrece al individuo la oportunidad de luchar por su propio óptimo bienestar y lograrlo. El individuo debe contar con personal de enfermería bien capacitado para vigilar sus programas de ejercicio, teniendo la certificación debida para los procedimientos de resucitación de emergencia. La (el) enfermera (o) debe tener, además, en el control de la presión arterial del paciente, de sus cambios electrocardiográficos y su fatiga, tanto antes del ejercicio, como durante y después del mismo.

La escala Borg viene con instrucciones escritas que indican lo siguiente:

"Usted participará ahora en una prueba de ejercicio, calificada.

Mientras camine o corra sobre la rueda de andar, estaremos midiéndole varias funciones fisiológicas. Deseamos también que trate de calcular la dificultad del trabajo; o sea, queremos que usted se califique en cuanto al grado de esfuerzo que usted perciba. Con decir esfuerzo percibido, hablamos de la cantidad total de esfuerzo y de fatiga física. No se preocupe por algún factor único, como sería el dolor de pierna, la falta de aliento, o la inclinación del trabajo. Mas 
bien trate de concentrarse en lo que es su sentir interno total del esfuerzo que esté realizando. Trate de hacer un cálculo lo más honesto y objetivo posible, sin menospreciar el grado de esfuerzo que sienta pero a la vez, tampoco sobreestimarlo. Solo trate, de calcularlo de la manera más precisa posible" (POLLOCK \& FILMORE, 1991).

Las instrucciones escritas se deben reforzar en voz alta. El concepto original del esfuerzo percibido fue desarrollado para los jóvenes. Cuando se aplicó la escala en el caso de personas de varias edades, se determinó que la misma relación lineal de intensidad de trabajo existía en todas las edades, pero que el pulso era cada vez más bajo por cada aumento en la edad (POLLOCK \& FILMORE, 1991).

Según FROELICHER (1987), la rehabilitación cardiaca y el uso de la escala Borg de esfuerzo percibido, utilizada para vigilar la fatiga, ayudaba a reestablecer el bienestar físico, psicológico y social a la vez que aminoraba las consecuencias negativas. Se encontró una relación importante entre la participación en la rehabilitación y las variables de salud, la calidad de vida, el ejercicio, la dieta y los cuidados personales (FROELICHER, 1987).

Por su lado, FROLICH (1992), uno de los líderes en la rehabilitación cardiaca, indicó que al ejercitar dos veces por semana, algunos individuos mostraran un aumento modesto en la salud cardiovascular. Para evitar el esfuerzo excesivo, el médico le define a cada individuo el pulso que deberá ser su meta. Agregado a esto, los individuos deben mantener un puntaje inferior a 13 en la escala Borg de esfuerzo percibido (GORDON \& GIBBONS, 1990).

Las exigencias del instrumento para con los sujetos serán completamente subjetivas y exigirán un mínimo de esfuerzo posterior al ejercicio (BORG, 1982). El cliente será el beneficiado principal de los resultados obtenidos durante el ejercicio y sabrá, de acuerdo a las instrucciones de su médico, cuándo empezar a ejercitar y cuándo parar su programa de ejercicio. Podrá visualizar y comparar la tasa inicial de su esfuerzo con su puntaje a lo largo de las semanas de ejercicio. El instrumento no es ni invasivo ni intruso. Sí presenta cierto riesgo mínimo que incluye el dolor en las articulaciones, cambios en el ritmo cardíaco y la muerte súbita (POLLOCK \& FILMORE, 1991). La tasa general de mortalidad reportada por los centros que utilizan la escala de esfuerzo percibido fue una muerte por cada 10.000 pruebas, y la tasa de complicaciones cardíacas graves fue de cuatro por 10.000 (POLLOCK \& FILMORE, 1991). La Cooper Clinic de Dallas, Texas informó tener una tasa de complicaciones de 0,8 por 10.000 evaluaciones (GORDON E GIBBONS, 1990).

Las limitaciones o dificultades encontradas en el uso de la escala de Borg incluyen las siguientes: 1) el uso de la escala con niños, ya sea porque no puedan leer las instrucciones o porque no entiendan los conceptos escritos; 2) el uso de la escala con mujeres embarazadas, dadas sus limitaciones físicas para realizar ejercicios; 3) las instrucciones y la escala están por escrito, lo cual impide a los 
analfabetas comprenderlas; y 4) su uso con personas cuyo estado psicológico o mental no les permite comprender o seguir las instrucciones.

\section{CONCLUSIÓN}

La escala Borg de esfuerzo percibido se puede usar en el hospital, o bien, fuera del ambiente hospitalario, para poder cuantificar los efectos de los programas de ejercicio. El cliente asume la responsabilidad al usar el instrumento. La prueba se puede utilizar al andar por la calle, al usar una rueda de andar, en bicicleta y con muchos otros estilos de ejercicio. El uso de la escala Borg de esfuerzo percibido, al detectar la fatiga precozmente, reducirá al mínimo la destrucción de la musculatura del corazón y promoverá así la buena salud.

\section{INSTRUMENT EVALUATION OF BORG'S PERCEIVED EXERTION SCALE IN CARDIAC REHABILITATION}

Borg's perceived exertion scale measures the extent of perceived exertion which a person experiences during exercise. The perceived exertion scale is used to adjust exercise intensity (workload) for predictions and prescriptions of exercise intensities in sports and medical rehabilitation (BORG, 1982). In addition, Borg's scale can be used in the athletic arena, space, industry, military settings, or under everyday circumstances. The concept of perceived exertion is a subjective rating, indicating the subject's opinion about the intensity of the work being performed (MORGAN, 1973). The task of exercising subjects is to assign a number (from 1-20) to represent the subjective sensation of the amount of work being performed. This is a valuable tool in human performance setting, where an important consideration is not "what the individual is doing, but what he thinks and is doing" (MORGAN, 1973).

UNITERMS: Borg's perceived exertion scale, cardiac rehabilitation, exercise rehabilitation

\section{REFERENCIAS BIBLIOGRAFICAS}

01. BLOEM, P.; GOESSENS, L.M.; ZAMPARO, P.; SACHER, M.; PAVIOTTI, R. de Prantero, P.E. Effects of order of presentation of exercise entensities and of sauna baths on perceived exertion during treadmill running. Rev.EUR.J.Appl.physioly, v.62, n. 3, p. 204-210. 1991. 
02. BORG, G. Psychophysical bases of perceived exertion (Las bases psicofísicas del esfuerzo percibido). J.Med.Sci.Sports Exercise, v. 14, n. 5, p. 377-381, 1982.

03. DUNBAR, C.; ROBERTSON, R,; BAUN, R.; BLANDIN, M.; METZ, K.; BURDETT, R.; GOSS, F. The validity of regulating exercise intensity by ratings of perceived exertion (La validez de controlar, por medio de puntajes de esfuerzo percibido, la intensidad del ejercicio). J.Med.Sci.Sports Exercise, v. 24, n. 1, p. 94-99, 1982.

04. FROELICHER, V.F. Exercise and the heart (el ejercicio y el corazón). 2.ed.Illinois: Year Book Medical Publishers, 1987.

05. FROHLICH, E.D. Preventive aspects of coronary heart disease (Los aspectos de la prevención de la enfermedad cardiaca). Pennsylvania: FA. Davis, 1990.

06. GORDON, M.; GIBBONS, L. The cooper clinic cardiac rehabilitation program (programa de Rehabilitación Cardiaca del Cooper Clinic). New York: Simon and Schuster, 1990.

07. MAHON, A.; MARSH, M. Reliability of the rating of perceived exertion at ventilatory threshold in children (La confiabilidad del puntaje de esfuerzo percibido en el umbral de ventilación en los niños). Int.J.Sports Med., v.13, n.8, p.567-571, 1992.

08. MORGAN, W. Psychological factors influencing perceived exertion (los factores psicológicos que influyen en la percepción de esfuerzo). J.Med.Sci. Sports Execise, v.5, n.2, p.97-103, 1973.

09. POLLOCK, D.; FILMORE, G. Prescription for programs of prevention and rehabilitation (Receta para los Programas de Prevención y Rehabilitación) 2.ed. Wisconsin: Word Publishing, 1991.

10. SKINNER, J.; HUTSLER, R.; BERGSTEINOVA, V.; BUSKIRK, E. The validity and reliability of a rating of perceived exertion (La validez y la confiabilidad de la calificación de esfuerzo percibido). J.Med.Sci.Sports Exercise, v. 6, n. 2, p. 994-996, 1973.

11. SUZUKI, S.; YOSHIKE, Y.; SUZUKI, M.; AKAHORI, TL.; HASEGAWA, A.; OKUBO, T. Inspiratory muscle training and respiratory sensation during treadmill exercise (El entrenamiento de los músculos de inspiración y la sensación respiratoria durante el ejercicio en la rueda de andar). Chest, v. 104, n. 1, p. 197-202, 1993.

12. WILSON, R.; OLDFIELD, W.; JONES, P. Effect of residence at altitude on the perception of breathlessness on return to sea level in normal subjects (En pacientes normales que regresan a nivel del mar, el efecto de la altitud de residencia sobre su percepción de falta de aliento). Clin.Sci., v.84, n.2, p.159-167, 1993. 


\section{Cuadro 1}

\section{ESFUERZO PERCIBIDO}

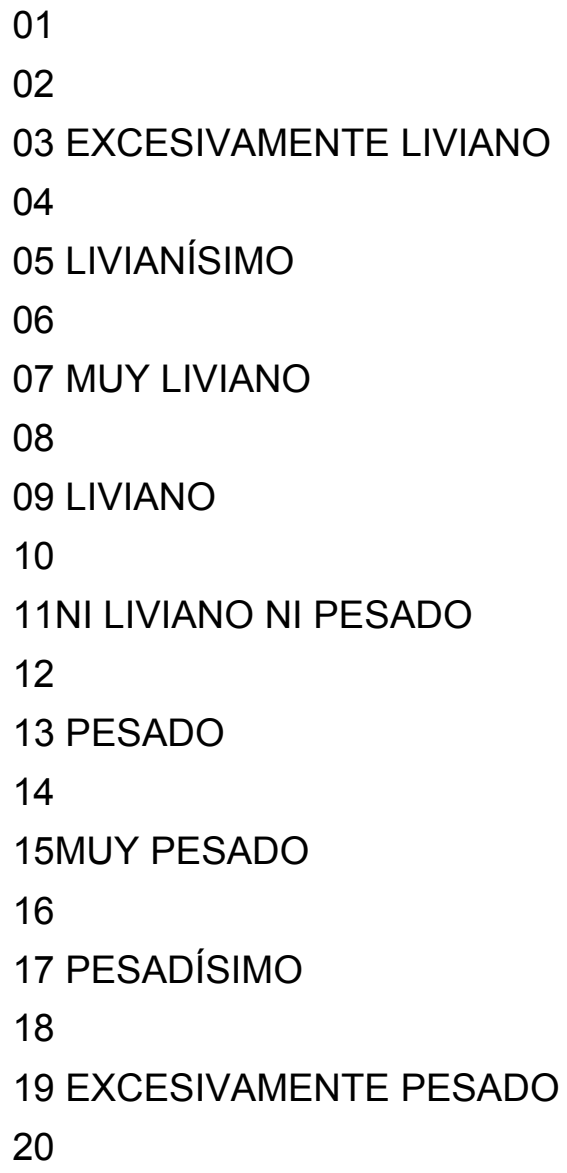

MORGAN, W. Psychological factors influencing perceived exertion (Los factores psicológicos que influyen en la percepción de esfuerzo). Journal of Medicine and Science in Sports and Exercise, v. 5, n. 2, p. 98, 1973. 
Figure 1

\section{PERCEIVED EXERTION}

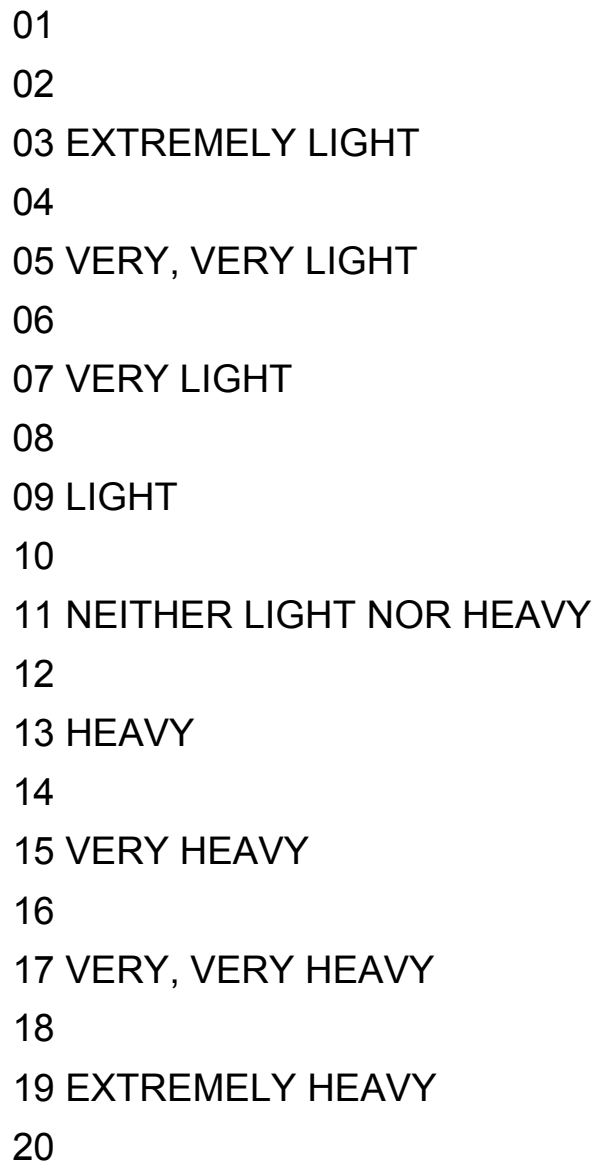

MORGAN, W. Psychological factors influencing perceived exertion. Journal of Medicine and Science in Sports, v. 5, n. 2, p. 98, 1973 